\title{
LOS FISICOS CONTRA LA CARRERA DE ARMAS NUCLEARES
}

\section{A CALL TO HALT THE NUCLEAR ARMS RACE}

Despite many efforts, conferences, and negotiations the nuclear arms race is accelerating. There are nowmore than fifty thousand nuclear weapons, some of which have yields a thousand times greater than the bomb that destroyed Hiroshima. This means some three tons T.VT equivalent for every person on earth. The counting of number of warheads and missiles is no longer relevant for security. On the contrary, the nuclear arms race diminishes security and brings us closer to nuclear holocaus.

Mankind's fate is in its own hands. The catastrophe of nuclear war can and must be prevented. The nuclear arms race must be reversed. It must be reversed now: there is no time to be lost. It can be reversed if people from all over the world work together for this common goal. Let us unite all voices to call for an immediate freeze of the nuclear arms race. This is a necessary first step towards nuclear disarmament. It is simple and comprehensive. People with different political and religious convictions, organizations and movements from many nations may work together to achieve it. The freeze should not be postponed until the conclusion of time consuming negotiations. We, physicists from all over the world, support the following appeal:

We call for an agreement to halt the testing, production and deployment of nuclear wapons and nuclear weapons delivery sustems. Meanwhile, no further nuclear weapons or delivery systems should be deployed anywhere.

\section{LA CARRERA DE ARMAS NLCLEARES}

A pesar de los muchos esfuerzos, conferencias y negociaciones la carrera de armas nucleares se esia acelerando. En estos momentos hay más de cincuenta mil armas nucleares, algunas de las cuales tienen campos de acción mil veces superior a la bomba que destruyó Hiroshima. Esto quiere decir que es equivalente a 3 toneladas de TNT por cada habitante de la tierra. El numero de torpedos y misiles no es nada tranquitizante para nuestra seguridad. Por el contrario. la carrera de armas nucleares disminuye nuestra seguridad y nos acerca cada vez más al holocausto nuclear.

El destino de la humanidad está en sus manos. Se debe prevenir la catástrofe de la guerra nuclear y se debe de hacer ahora, ya que no hay tiempo que perder. Se podria prevenir si todas las personas del mundo trabajasen juntas para la obtención de este objetivo común. Vamos a unir todas las voces para pedir una inmediata congelación de la carrera de armas nucleares. Esto es un primer paso necesario hacia el desarme nuclear. Es muy sencillo. Las personas de diferente conviccion politica y religiosa y las organizaciones y movimientos de muchas naciones deben trabajar juntos para conseguirlo. No se debe posponer la congelación a la fina lización de las negociaciones. Nosotros, los físicos de todo el mundo apoyamos la siguiente llamada:

Pedimos que se llegue a un acuerdo para detener las pruebas, producción y despliegue de armas nucleares y de los sistemas de reparto de las mismas. Mientras tanto, en ningún lugar debe continuar el despliegue de más armas nucleares y sistemas de distribución.

First signatories include: H. ALFVEN (Sweden) • J. ALTSHULER (Cuba) • E. AMALDI (Italy) • D. AMATI $($ taly) $\bullet$ N.G. ANTONIOU (Greece) • A. ARIMA (Japan) • D.R. BATES (N.Irel) • N. BASOV (USSR) I.S. BELL (UK) • D.R. BES (Argent.) • H.A. BETHE (LSA) • F. CALOGERO (Italy) • H.G.B. CASIMIR (Netherl.) • G. CHARPAK (France) • P.A. CHERENKOV (URSS) • W. CHINOWSKY (USA) • S.G. COHEN (Israel) • J.W. CRONIN (USA) - F. DYSON (USA)・R. FEYNMAN (USA)・R. FIESCH! (Italy) • I.M. FRANK (URSS) - Gr. CHIKA (Romania)) - S. GLASHOW (USA) - P. KAPITZA (URSS) - A. KASTLER (France) - T.W.B. KIBBLE (UK) • R. KUBO (Japan) • F. LALOE (France) - K.V. LAURIKAINEN (Finland) • L. EEDERMAN (USA) - M.A. MARKOV (USSR) • J.V. NARLIKAR (India) • Ph. NOZIERES (France) • R. PEIERLS $($ UK) - J. PNIEWSKI (Poland) - A.M. PROKHOROV (USSR) - F. RAVNDAL (Norway) • M. RESS (UK) - T. REGGE (Italy) • M. ROSENBLUTH (USA) • A. ROSENFELD (USA)・C. RUBBIA (Italy) • W.A. RUNCIMAN (Australia) • M. RYLE (UK) • A. SALAM (UK) • N.C. SANCHEZ (Spain) • F.D. SANTOS (Portugal) - K. SIEGBAHN (Sweden)) • L. SOSNOWSKI (Poland) • T.D. SPEARMAN (Ireland) • P. STEIN (USA) • J. STEINBERGER (USA) - I. TALMI (Israel) • V.L. TELEGDI (Switzerland) • W. THIRRING (Austria) • J. TIOMNO (Brazil) - S. TITEICA (Romania) - I.T. TODOROV (Bulgaria)) • H.J. TREDER (Germ. Dem. Rep.) - A. UHLMANN (Germ. Dem. Rep.) • S. WEINBERG (USA) • V.F. WEISSKOPF (USA) • Ch. WETTERICH (Germ. Fed. Rep.) - YA.B. ZELDOVICH (USSR) - A. ZICHICHI (laty). 